\title{
The Routing Continuum from Shortest-path to All-path: A Unifying Theory
}

\author{
Yanhua Li, Zhi-Li Zhang, Daniel Boley \\ Department of Computer Science \& Engineering, University of Minnesota, Twin Cities \\ Email: \{yanhua,zhzhang,boley\}@cs.umn.edu
}

\begin{abstract}
Routing is a critical operation in networks. In the context of data and sensor networks, routing strategies such as shortest-path, multi-path and potential-based ("all-path") routing have been developed. Based on the connection between routing and flow optimization in a network, in this paper we develop a unifying theoretical framework by considering flow optimization with mixed (weighted) $L_{1} / L_{2}$-norms. We obtain a surprising result: as we vary the trade-off parameter $\theta$, the routing graphs induced by the optimal flow solutions span from shortest-path to multi-path to all-path routing - this entire sequence of routing graphs is referred to as the routing continuum. Our theory subsumes the earlier results showing the shortest path and allpath routing can be obtained from $L_{1}$ and $L_{2}$ flow optimization, respectively. We also develop an efficient iterative algorithm for computing the entire routing continuum. Several generalizations are also considered, with applications to traffic engineering and wireless sensor networks.
\end{abstract}

\section{INTRODUCTION}

Routing is a critical operation in many types of networks from communication networks to transportation networks. For instance, in modern IP-based data networks, shortest path routing is most commonly used. In traditional telecommunication networks, dynamic alternative routing strategies that employ paths that are longer than shortest paths have been also proposed to reduce call blocking probabilities (see, e.g., [1], [16]). In wireless networks, due to the unstable channel characteristics, using a single "shortest" path (e.g., with link quality as link weights) for routing is often not the best choice; routing strategies that go beyond shortest path routing (see, e.g., [4], [15], [18], [25] and references therein) using multiple paths are often more effective. In the other extreme, in wireless sensor networks - due to their power and other resource constraints - potential-based routing [19] has been proposed, where the source essentially utilizes all (eligible) paths to transmit data to the destination. In [19], it is shown that such "all-path" routing minimizes the total energy dissipation of routing and thus maximizes the network lifetime. Clearly, what routing strategies to employ in a network hinges on what objectives are important in practice, therefore should be optimized. However, from a theoretical perspective, when using multi-path routing that goes beyond a single shortest path, two questions arise: i) what set of paths should be used for routing? and ii) how traffic should be split (and merged) at any node along the multiple paths, especially when the paths are not all disjoint?

This work was supported in part by the NSF grants CNS-1017092 and IIS-0916750, the DTRA grant HDTRA1-09-1-0050.
In addressing these questions, in this paper we consider routing as flow optimization in a network with mixed (weighted) $L_{1} / L_{2}$-norms as the objective. This formulation is inspired by the earlier results where it has been shown that shortest path routing can be derived from network flow optimization with $L_{1}$ norm [30], whereas potential-based, "all-path" routing can be derived from network flow optimization with $L_{2}$-norm objective [16], [19]. Intuitively, the mixed $L_{1} / L_{2}$-norm objective can be interpreted as a tradeoff between the latency and energy dissipation of paths used for routing (collectively, the paths form a routing graph): shorter paths lead to better routing with low latency, while diffusing traffic along more paths generally reduces energy dissipation. Using this formulation, we obtain a surprising result: as we vary the trade-off parameter $\theta$, the routing graphs induced by the optimal flow solutions span from the shortest-path routing to multi-path routing with increasing path lengths to the potential-based ("all-path") routing - this entire (finite) sequence of routing graphs is referred to as the routing continuum. Our theory therefore subsumes the earlier $L_{1}$ and $L_{2}$ network flow optimization results [16], [30] as two extreme points in the entire routing continuum.

Furthermore, by considering the dual of the mixed $L_{1} / L_{2}$ norm network flow optimization problem, we develop an efficient iterative algebraic process as well as algorithms for identifying precisely the boundary conditions separating the finite sequence of routing graphs, and for computing the entire routing continuum and optimal flow solutions $X^{*}(\theta)$ for any $\theta \geq 0$. In particular, $X^{*}(\theta)$ specifies how traffic should be split and merged in the induced routing graph. We also generalize the theory to account for multiple flows (traffic demands), link capacity constraints and heterogeneous $L_{1} / L_{2}$ link weights, with applications to traffic engineering and wireless sensor networks. For instance, given a set of link weights and traffic demands on a network, our theory can be used to find the "best" routing graph (i.e., the best mix of shorter and longer paths) that minimizes the overall maximum link utilization.

In summary, our contributions are i) we develop a unifying theory using mixed $L_{1} / L_{2}$-norm network flow optimization and show that it can generate the entire routing continuum from shortest-path to "all-path" routing; ii) we develop an efficient iterative process for computing the entire routing continuum and optimal flow solutions $X^{*}(\theta)$ for any $\theta \geq 0$; iii) the basic theory is further generalized to account for multiple flows (traffic demands), link capacity constraints 
and heterogeneous $L_{1} / L_{2}$ link weights, with applications to traffic engineering and wireless sensor networks. Last but not the least, while we focus on network routing in this paper, we believe that our results can be applied to many other applications where the problems can be cast in terms of flows in a network.

The remainder of the paper is organized as follows. In Section II we introduce the basic notations and re-state the known $L_{1}$ and $L_{2}$ flow optimizations using our notations. In Section III the general theory and results using the mixed $L_{1} / L_{2}$-norm flow optimization are established, and the iterative computation process and algorithms are described in Section IV. In Section V, we consider several generalizations, with applications to traffic engineering and wireless sensor networks. Section VI discusses the related work, and the paper is concluded in Section VII.

\section{Shortest Path and Potential-Based "All-Path" ROUTING AS NETWORK Flow OPTIMIZATION}

In this section, we first introduce the basic notations that will be used throughout the paper. Then, we illustrate how shortest path routing and potential-based "all-path" routing can be formulated as the flow optimization problems in a network using metric norms (on the flow space). More specifically, the shortest path routing results from minimizing the (weighted) $L_{1}$-norm of flows between a given source-destination pair in a network, whereas the potential-based, "all-path" routing results from minimizing the corresponding $L_{2}$-norm.

\section{A. Network and Flows: Basic Notations}

We represent a $n$-node network as an undirected, weighted graph, $G=(V, E, W)$, where $V=\{1,2, \ldots, n\}$ is the set of vertices, $E$ is the set of edges, and each edge $(i, j) \in E$ is assigned a positive weight $w_{i j}$. As $G$ is undirected, $(i, j)$ and $(j, i)$ represent the same edge in $E$, and $w_{i j}=w_{j i}>0$. Define $w_{i j}=0$ if $(i, j) \notin E$, then the weight matrix $W=\left[w_{i j}\right]$ is symmetric. In particular, if all edges have a unit weight, i.e., $W$ is a $0-1$ matrix, then $G$ represents a simple graph, and $W$ is the corresponding adjacency matrix.

Let $d=[s, t], s, t \in V, s \neq t$, denote a source-destination (or source-sink) pair in the network $G$. A flow of $I^{(d)}$. unit amount that flows from source $s$ to destination $t$ is mathematically defined as a function, $X^{(d)}: V \times V \rightarrow \mathbb{R}^{+}$ $\left(\mathbb{R}^{+}\right.$is the set of non-negative real numbers), satisfying the following constraints:

$$
\text { along one direction: }
$$

$$
\text { if } X_{i j}^{(d)}>0 \text { then } X_{j i}^{(d)}=0 \text {, }
$$$$
\text { if }(i, j) \notin E \text { then } X_{i j}^{(d)}=0 \text {, }
$$

along network edges:

$$
I^{(d)}+\sum_{k=1}^{n} X_{k s}^{(d)}=\sum_{j=1}^{n} X_{s j}^{(d)}
$$

flow conservation at $s: \quad I^{(d)}+\sum_{k=1}^{n} X_{k s}^{(d)}=\sum_{j=1}^{n} X_{s j}^{(d)},(3)$

intermediate node $i \neq s, t: \quad \sum_{k=1}^{n} X_{k i}^{(d)}=\sum_{j=1}^{n} X_{i j}^{(d)}$,

$$
\text { at destination } t: \quad \sum_{k=1}^{n} X_{k t}^{(d)}=\sum_{j=1}^{n} X_{t j}^{(d)}+I^{(d)}
$$

Note that in this flow definition, for each (undirected) edge $(i, j) \in E$, both $X_{i j}^{(d)}$ and $X_{j i}^{(d)}$ are defined, and the constraint in eq.(1) states that if $X_{i j}^{(d)}>0$, then $X_{j i}^{(d)}=0$; or if $X_{j i}^{(d)}>$
0 , then $X_{i j}^{(d)}=0$. It is possible that for $(i, j) \in E$, both $X_{i j}^{(d)}=X_{j i}^{(d)}=0$. In particular, by the constraint in eq.(2), $X_{i j}^{(d)}=X_{j i}^{(d)}=0$ for $(i, j) \notin E$. The flow constraints in eqs.(3)-(5) state that a (net) amount of $I^{(d)}$ units of flow is injected at source $s$, and the same amount is removed from destination (sink) $t$, while the amount of flow entering any intermediate node $i$ is the same as the amount leaving the node.

Given a flow $X^{(d)}$ between a source-destination pair $d=$ $[s, t]$, it induces an oriented (or directed) sub-graph of $G$, $G_{X^{(d)}}=\left(V_{X^{(d)}}, E_{X^{(d)}}\right)$, where an $\operatorname{arc}\langle i, j\rangle \in E_{X^{(d)}}$ and $i, j \in V_{X^{(d)}}$ if and only if $X_{i j}^{(d)}>0$. As a directed acyclic graph (DAG) between $s$ and $t, G_{X^{(d)}}$ represents the routes used to route the flow $X^{(d)}$ (of $I^{(d)}$ units) from source $s$ to destination $t$, and we refer to it as the routing graph for the flow $X^{(d)}$. When $G_{X^{(d)}}$ consists of more than a single path between $s$ and $t$, then $X_{i j}^{(d)}$ indicates how much flow is routed along the edge (arc) $\langle i, j\rangle$. In general, the flow may be split or merged $^{1}$ at nodes in $G_{X^{(d)}}$, and routed along different paths between $s$ and $d$. We will use $\mathcal{F}^{(d)}$ to denote the collection of flows, i.e., all functions that satisfy eqs.(2)-(5).

In the next two subsections we will use two well-known results [16], [30] to illustrate that certain common routing strategies, namely, shortest path routing and potential-based, "allpath" routing, can be derived by minimizing the (weighted) $L_{1}$-norm and $L_{2}$-norm, respectively, of flows between a given source-destination pair in a network. In Section III we will generalize these results and establish that by minimizing flows using mixed $L_{1}$-norm and $L_{2}$-norm, we can generate a continuum of routing strategies, resulting in a sequence of routing graphs with varying numbers of paths of differing costs selected, from the shortest paths to all paths (between a sourcedestination pair).

\section{B. Shortest-Path Routing and $L_{1}$-norm Flow Optimization}

Without loss of generality, unless otherwise specified, we assume that $s=1$ and $t=n$, and $I^{(d)}=1$. For clarity of notation, we drop the superscript $d$ from $X^{(d)}$. In other words, the flow $X$ (as a function) is equivalently specified by a set of $n^{2}$ variables, $X_{i j}$ 's, $1 \leq i, j \leq n$.

Consider the following $L_{1}$-norm network flow optimization problem, which can be solved using linear programming (LP). $L_{1}$-norm Network Flow Optimization ( $L_{1}$ Primal):

$$
\min _{X} \sum_{i=1}^{n} \sum_{j=1}^{n} w_{i j} X_{i j}
$$

subject to the flow conservation constraints eqs.(2)-(5), which

${ }^{1}$ The flow definition implicitly assumes that flows are "infinitely divisible fluid" - they can be split and merged arbitrarily at any node of the network, as long as the above flow conservation constraints are met. This mathematical definition of network flows thus provides an idealized (fluid) abstraction of, e.g., traffic demands routed from a source to a destination in a communication network, or commodities transported from a source to a destination in a transportation network, and so forth. 
are more compactly represented below using $X_{i j}$ 's:

$$
\sum_{j:(i, j) \in E} X_{i j}-\sum_{k:(k, i) \in E} X_{k i}= \begin{cases}1 & \text { if } i=1 \\ 0 & \text { if } i=2, \ldots, n-1 \\ -1 & \text { if } i=n,\end{cases}
$$

and $X_{i j} \geq 0,1 \leq i, j \leq n$.

Note that eq.(7) is a combination of the constraints in eqs.(2)-(5) and the constraints in eq.(8) are more relaxed than the constraints in eq.(1) - they are used mostly for their convenience in deriving the dual optimization problem. However, consider any $X$ that satisfies the flow conservation constraint eq.(7). Suppose that there exists an edge $(i, j) \in E$ such that $0<X_{j i} \leq X_{i j}$. Define $X_{i j}^{\prime}=X_{i j}-X_{j i}$ and $X_{j i}^{\prime}=0$. It is easy to see that $X^{\prime}$ satisfies all constraints in eqs.(1)-(5). Hence $X^{\prime}$ is a flow. Furthermore, $\sum_{i=1}^{n} \sum_{j=1}^{n} w_{i j} X_{i j}^{\prime}<\sum_{i=1}^{n} \sum_{j=1}^{n} w_{i j} X_{i j}$. Hence without loss of generality, when considering the optimization in eq.(6), we can restrict ourselves to $X$ 's that are flows, i.e., $X \in \mathcal{F}$. Thus we can re-state the optimization in eq.(6) as

$$
\min _{X \in \mathcal{F}} \sum_{i}^{n} \sum_{j}^{n} w_{i j} X_{i j}
$$

In other words, the optimization solution to eq.(6) is the flow that minimizes the weighted $L_{1}$-norm.

To show that the optimal solution to this $L_{1}$-norm network flow optimization gives rise to the shortest-path routing, we consider its dual, stated below in terms of the Lagrange multipliers $-U_{i}$ 's (corresponding to the flow conservation constraints eq. $\left.(7)^{2}\right)$ :

Dual of $L_{1}$-norm Network Flow Optimization ( $L_{1}$ Dual):

$$
\max _{U} U_{1}
$$

subject to $U_{n}=0$ and $U_{i}-U_{j} \leq w_{i j}, \forall(i, j) \in E$.

Let $X^{*}$ denote the optimal flow solution to the primal problem eq.(6), and $U^{*}$ the optimal solution to the dual problem. The duality and complementary slackness give us the following relations between $X_{i j}^{*}$ 's and $U_{i}^{*}$ 's ( $c f$. Lemma 1 in [30]):

$$
\begin{aligned}
\text { if } X_{i j}^{*} & >0 \text {, then } U_{i}^{*}-U_{j}^{*}=w_{i j} \text {; } \\
\text { and if } X_{i j}^{*} & =0 \text {, then } U_{i}^{*}-U_{j}^{*}<w_{i j} \text {. }
\end{aligned}
$$

Using these relations, the authors in [30], show that the optimal solution to the dual problem, $U_{i}^{*}$ 's, have the following properties ( $c f$. Theorem 1 and its proof in [30]):

LEMMA 1. Let $P$ be a path from node 1 to node $n$. If for each edge (arc) $\langle i, j\rangle \in P, U_{i}^{*}-U_{j}^{*}=w_{i j}$, then $P$ is a shortest path from node 1 to node $n$ (with respect to the weights $w_{i j}$ 's), and $U_{1}^{*}=\sum_{\langle i, j\rangle \in P} w_{i j}$. Alternatively, if $Q$ is a path from node 1 to node $n$ that is not a shortest path, then $U_{1}^{*}<$ $\sum_{\langle i, j\rangle \in Q} w_{i j}$.

\footnotetext{
${ }^{2}$ Note that our Lagrange multipliers are negatives of those used in the "Dual Shortest Path Formulation (D-SP)" in [30], p. 3.
}

The above lemma implies that for any node $i$ on a shortest path, $U_{i}^{*}$ is the shortest-path distance from node $i$ to node $n$ (the destination). Furthermore, the optimal flow $X^{*}$ is only routed along the shortest paths between source 1 and destination $n$. In other words, the resulting routing graph $G_{X^{*}}$ is the DAG formed by the shortest paths from 1 to $n$ only. When there are multiple shortest paths between 1 and $n, X_{i j}^{*}$ specifies the amount of flow carried on the edges of node $i$ that are on the shortest paths, thus how the flow should be split among multiple shortest path at node $i$.

\section{Potential-based ("All-path") Routing and $L_{2}$-norm Flow Optimization}

We now consider the following (weighted) $L_{2}$-norm network flow optimization problem:

$L_{2}$-norm Network Flow Optimization ( $L_{2}$ Primal):

$$
\min _{X \in \mathcal{F}} \sum_{i=1}^{n} \sum_{j=1}^{n} w_{i j} X_{i j}^{2}
$$

To show that the optimal solution to this $L_{2}$-norm network flow optimization gives rise to the potential-based, "all-path" routing, we again consider its dual, stated below in terms of the Lagrange multipliers $U_{i}$ 's (where for convenience we have used $-2 U_{i}$ 's as the multipliers for the flow conservation constraints eq.(7)):

Dual of $L_{2}$-norm Network Flow Optimization ( $L_{2}$ Dual):

$$
\max _{U} U_{1}-\frac{1}{2} \sum_{i=1}^{n} \sum_{j: U_{i}>U_{j}} \frac{\left(U_{i}-U_{j}\right)^{2}}{w_{i j}} .
$$

subject to $U_{n}=0$.

Let $X^{*}$ denote the optimal flow solution to the primal problem eq.(13), and $U^{*}$ the optimal solution to the dual problem. The duality and complementary slackness give us the following relations between $X_{i j}^{*}$ 's and $U_{i}^{*}$ 's: for any edge $(i, j) \in E$,

$$
\begin{aligned}
& \text { if } U_{i}^{*}>U_{j}^{*} \text {, then } X_{i j}^{*}=\frac{U_{i}^{*}-U_{j}^{*}}{w_{i j}}>0 ; \\
& \text { and if } U_{i}^{*} \leq U_{j}^{*} \text {, then } X_{i j}^{*}=0 .
\end{aligned}
$$

If we treat $w_{i j}$ as the resistance on edge $(i, j) \in E$, then the relation eq.(15) gives us precisely Ohm's law [16], and $U_{i}^{*}$ is the voltage (potential) at node $i$ when a unit of current is injected at source node 1 and removed at sink node $n$ (and grounded with $U_{n}^{*}=0$ ). For any $(i, j) \in E$, if $U_{i}^{*}>U_{j}^{*}$, then the current $I_{i j}$ flowing from node $i$ to node $j$ along edge $(i, j)$ is exactly $X_{i j}^{*}$, as $I_{i j}=\left(U_{i}^{*}-U_{j}^{*}\right) / w_{i j}=X_{i j}^{*}>0$. (In a electrical network, the reverse current flow, i.e., the current from node $j$ to node $i$ is defined as $I_{j i}:=-I_{i j}=-X_{i j}^{*}<0$.) Hence the optimal solution to the dual problem eq.(14), $U^{*}$, is a potential function (the voltage potential in the electrical network $G$ ): $U_{i}^{*}$ is the voltage potentialfrom node $i$ to destination node $n$ (ground).

For $(i, j) \in E$, define $a_{i j}:=1 / w_{i j}$, the conductance on edge $(i, j)$, and for $(i, j) \notin E, a_{i j}=0$. From the 
flow conservation constraints (or directly by solving the dual optimization problem eq.(14)), we see that

$$
U_{i}^{*}=\left\{\begin{array}{ll}
\sum_{j=1}^{n} \frac{a_{i j}}{\sum_{k} a_{i k}} U_{j}^{*}+\frac{1}{\sum_{k} a_{i k}} & \text { if } i=1 \\
\sum_{j=1}^{n} \frac{a_{i j}}{\sum_{k} a_{i k}} U_{j}^{*} & \text { if } i=2, \ldots, n-1 \\
0 & \text { if } i=n
\end{array},\right.
$$

which gives the Kirchhoff's law for voltage in an electrical network. The dual problem eq.(14) gives us the Dirichlet principle [16]: the voltage potentials, $U^{*}$, taken within the electrical network $G$ minimizes the total energy dissipation. Likewise, the $L_{2}$-norm flow optimization problem also has a physical interpretation (Thompson's Principle [16]): among all flows $X \in \mathcal{F}$, the optimal (current) flow, $X^{*}$, minimizes the energy dissipation in the (electrical) network.

This connection between currents (and voltage) in electrical networks and $L_{2}$-norm network flow optimization is well known in the literature (see, e.g., [16]), and gives rise to potential-based ("all-path") routing (or "stochastic routing") in communication and wireless sensor networks [16], [19]. Using the relations eq.(15) and eq.(16), it is easy to see that for any path $P$ from node 1 (source) to node $n$ (destination) in the network $G$, the (current) flow along $P$ is nonzero (i.e., $X_{i j}^{*}>0, \forall\langle i, j\rangle \in P$ ) if and only if the potential (voltage) at any node $i$ along the path from node 1 to node $n$ is strictly decreasing (i.e., $\forall\langle i, j\rangle \in P, U_{i}^{*}>U_{j}^{*}$ ). Hence the routing graph $G_{X^{*}}$ induced by the optimal flow to the $L_{2}$-norm flow minimization problem is a DAG consisting of any path from source node 1 to destination node $n$ with strictly decreasing potentials - that is what we also refer to the potential-based routing as "all-path" routing. Moreover, Ohm's law specifies how flows along the paths are split - proportional to the potential difference along an edge and inverse to the resistance of the edge, namely, $X_{i j}^{*}=\left(U_{i}^{*}-U_{j}^{*}\right) / w_{i j}$.

\section{MIXED $L_{1}$ AND $L_{2}$-NORM NETWORK FLOW OPTIMIZATION AND THE ROUTING CONTINUUM}

The results in the previous section show that the optimal flows that minimize the (weighted) $L_{1}$-norm and $L_{2}$-norm in a network yield the shortest path and (potential-based) "all-path" routing, respectively. Intuitively, if we treat $w_{i j}$ as "delay" 3 on each link $(i, j)$, then the $L_{1}$-norm minimization produces an optimal flow routing that minimizes the total delay; whereas the $L_{2}$-norm minimization produces an optimal flow routing that minimizes the total energy dissipation (treating $w_{i j}$ as the resistance of link $(i, j)$ ). This gives rise to a natural question: can we generate other routing strategies between these two extremes, e.g., routing using shortest paths as well as secondshortest paths, via network flow optimization with respect to some other forms of cost metrics? In particular, can these routing strategies be derived by trading off the total delay (the $L_{1}$-norm) and the total energy (the $L_{2}$-norm)? This leads us

\footnotetext{
${ }^{3} \mathrm{Or}$ in a physical meaning that is more consistent with the $L_{2}$-norm interpretation, the "(resistive or fricative) force" needed to move a unit of flow across link $(i, j)$.
}

to posing the following mixed $L_{1}$ - and $L_{2}$-norm network flow optimization problem, where $\theta \geq 0$.

Mixed $L_{1}$ - and $L_{2}$-norm Network Flow Optimization (Primal):

$$
\min _{X \in \mathcal{F}} \sum_{i=1}^{n} \sum_{j=1}^{n} w_{i j} X_{i j}^{2}+2 \theta \sum_{i=1}^{n} \sum_{j=1}^{n} w_{i j} X_{i j} .
$$

Consider the dual of this flow optimization problem, which can be derived and expressed in terms of the Lagrange multipliers $-2 U_{i}$ 's as follows:

Mixed $L_{1}$ - and $L_{2}$-norm Network Flow Optimization (Dual):

$$
\begin{aligned}
& \max _{U} U_{1}-\frac{1}{2} \sum_{i} \sum_{j: U_{i}-U_{j}>\theta w_{i j}} \frac{\left(U_{i}-U_{j}-\theta w_{i j}\right)^{2}}{w_{i j}} \\
& \text { s.t. } U_{n}=0 .
\end{aligned}
$$

Let $X^{*}(\theta)$ be the optimal solution to the primal problem eq.(18), and $U^{*}(\theta)$ the optimal solution to the dual problem eq.(19). Again from the duality and complementary slackness, we have the following relations between $X^{*}(\theta)$ and $U^{*}(\theta)$ :

$$
X_{i j}^{*}(\theta)= \begin{cases}\frac{U_{i}^{*}(\theta)-U_{j}^{*}(\theta)}{w_{i j}}-\theta & \text { if } U_{i}^{*}(\theta)-U_{j}^{*}(\theta)>\theta w_{i j} \\ 0 & \text { if } U_{i}^{*}(\theta)-U_{j}^{*}(\theta) \leq \theta w_{i j}\end{cases}
$$

Clearly, $\theta=0$ gives us the $L_{2}$-norm network flow optimization. In the following we will show that for sufficiently large $\theta$, the routing graph induced by the optimal solution to eq.(18) gives the same shortest path DAG as the $L_{1}$-norm flow optimization. In other words, for sufficiently large $\theta$, the optimal solution to eq.(18) yields the shortest path routing. Furthermore, for $\theta$ in between, the optimal solution to eq.(18) yields a continuum of routing graphs with the "all-path" and shortest-path DAGs as two extremes in the continuum.

Fix $\theta \geq 0$, and let $G_{X^{*}(\theta)}$ denote the routing graph (DAG) induced by the optimal flow solution $X^{*}(\theta)$ to eq.(18), i.e., for any edge $(i, j) \in E$, the $\operatorname{arc}\langle i, j\rangle$ is included in $G_{X^{*}(\theta)}$ if and only $X_{i j}^{*}(\theta)>0$. We use $P \in G_{X^{*}(\theta)}$ to denote a path $P$ from node 1 (source) to node $n$ (destination) where the flow along this path is nonzero, i.e., for any $\langle i, j\rangle \in P, X_{i j}^{*}(\theta)>0$. We have the following lemma:

LEMMA 2. Consider any path $P \in G_{X^{*}(\theta)}$, and $Q$ be any path from node 1 to node $n$. The following holds:

$$
\theta \sum_{\langle i, j\rangle \in P} w_{i j}<U_{1}^{*}(\theta) \leq(\theta+1) \sum_{\langle i, j\rangle \in Q} w_{i j} .
$$

Proof : For any $\langle i, j\rangle \in P$, since $X_{i j}^{*}(\theta)>0$, from eq.(21) we have $U_{i}^{*}(\theta)-U_{j}^{*}(\theta)=\theta w_{i j}+w_{i j} X_{i j}^{*}(\theta)$. Therefore $\sum_{\langle i, j\rangle \in P}\left(U_{i}^{*}(\theta)-U_{j}^{*}(\theta)\right)=\sum_{\langle i, j\rangle \in P}\left(\theta w_{i j}+w_{i j} X_{i j}^{*}(\theta)\right)$. Hence

$$
U_{1}^{*}(\theta)=\sum_{\langle i, j\rangle \in P}\left(\theta w_{i j}+w_{i j} X_{i j}^{*}(\theta)\right)>\theta \sum_{\langle i, j\rangle \in P} w_{i j}
$$

as $X_{i j}^{*}>0$ for any $\langle i, j\rangle \in P$. On the other hand, for any $\langle i, j\rangle \in Q$, from eq.(21) we have $U_{i}^{*}(\theta)-U_{j}^{*}(\theta) \leq \theta w_{i j}+$ 
$w_{i j} X_{i j}^{*}(\theta)$, where the inequality holds when $X_{i j}^{*}(\theta)=0$. Summing up along all edges $\langle i, j\rangle \in Q$, we have

$$
U_{1}^{*}(\theta) \leq \sum_{\langle i, j\rangle \in Q}\left(\theta w_{i j}+w_{i j} X_{i j}^{*}(\theta)\right) \leq(\theta+1) \sum_{\langle i, j\rangle \in Q} w_{i j}
$$

where the last inequality follows from the fact that $X_{i j}^{*} \leq$ 1. Combining this and the inequality in eq.(23) proves the lemma.

From Lemma 2, the following holds for any $\theta>0$,

$$
\sum_{\langle i, j\rangle \in P} w_{i j}<\left(1+\theta^{-1}\right) \min _{Q} \sum_{\langle i, j\rangle \in Q} w_{i j} .
$$

Using this Lemma, we establish the following theorem.

THEOREM 1 (Routing Continuum). Let $\mathcal{R}(0)$ denote the (potential-based) "all-path" routing graph in Section II-C, namely, the routing graph induced by the optimal $L_{2}$-norm flow $X^{*}(0)$, the optimal solution to eq.(18) with $\theta=0$. Let $\mathcal{P}$ denote the collection of all paths (with nonzero flow), $P \in$ $\mathcal{R}(0)$, from source node 1 to destination $n$. Sort and group the paths based on their length, i.e., $|P|:=\sum_{\langle i, j\rangle \in P} w_{i j}$, which yields a partition (equivalent classes) of $\mathcal{P}: \mathcal{P}_{1}, \ldots, \mathcal{P}_{M}$, where $\mathcal{P}_{m}=\left\{P \in \mathcal{R}(0):|P|=L_{m}\right\}, m=1, \ldots, M$, and $L_{1}<\ldots<L_{M}$. Clearly $L_{1}$ is the length of the shortest paths.

For $\theta>0$, let $\mathcal{R}(\theta)$ denote the routing graph induced by the optimal flow $X^{*}(\theta)$, the solution to the mixed $L_{1}$ - and $L_{2}$ norm flow optimization problem. Then for $\left(L_{m}-L_{1}\right) / L_{1}<$ $\theta^{-1} \leq\left(L_{m+1}-L_{1}\right) / L_{1}, m=1, \ldots, M$ (here we define $\left.L_{M+1}=\infty\right)$, we have

$$
\mathcal{R}(\theta) \subseteq \cup_{k=1}^{m} \mathcal{P}_{k}
$$

In other words, $\mathcal{R}(\theta)$ contains the paths of length at most $L_{m}$.

Proof : We prove by contradiction. Given any $m, m=$ $1, \ldots, M$, and $\theta>0$ where $\left(L_{m}-L_{1}\right) / L_{1}<\theta^{-1} \leq$ $\left(L_{m+1}-L_{1}\right) / L_{1}$, suppose there exists $P \in \mathcal{R}(\theta)$ such that $|P|>L_{m}$ (thus $|P| \geq L_{m+1}$ ). From Lemma 2, the length of any path in the routing graph $\mathcal{R}(\theta)$ used to route the optimal flow $X^{*}(\theta)$ is less than $\left(1+\theta^{-1}\right) L_{1} \leq L_{m+1}$. This leads to a contraction.

Theorem 1 states as $\theta$ increases from 0 to $\infty$, or equivalently $\theta^{-1}$ decreases to 0 , longer paths in $\mathcal{R}(0)$ are pruned, yielding a "sparser" routing graph $\mathcal{R}(\theta)$ that contains only paths of length less than $\left(1+\theta^{-1}\right) L_{1}$. In fact, there are a finite sequence of routing graphs $\mathcal{R}_{m}, 1 \leq m \leq M$, where $\mathcal{R}_{m}$ only contains paths of length at most $L_{m}$. We refer to this sequence of routing graphs as the routing continuum. In the next section we will present an algorithm for explicitly constructing the routing continuum, and in particular, for computing the optimal flow solution, $X^{*}(\theta)$, which specifies how the optimal flow is routed among the paths in $\mathcal{R}_{m}$.

\section{Computing the Routing Continuum}

In this section we describe an efficient algorithm for computing the routing continuum and the associated optimal flow $X^{*}(\theta)$ for all $\theta$ 's, and use two simple examples to illustrate the algorithm and results obtained thereof.

\section{A. Routing Continuum Algorithm (RCA)}

We introduce an iterative process for computing the routing continuum and the optimal flow $X^{*}(\theta)$, starting with $\theta=0$, where each step involves solving a set of linear equations in $U_{i}^{*}(\theta)$ 's.

For any $\theta$, let $\mathcal{R}(\theta)=(V(\theta), E(\theta))$ denote the routing graph induced by $X^{*}(\theta)$, a subgraph of $G=(V, E)$, where $(i, j) \in \mathcal{R}(\theta)$ if and only if $X_{i j}^{*}(\theta)>0$. In the following, we will treat $\mathcal{R}(\theta)$ as an undirected graph. Hence an edge $(i, j) \in \mathcal{R}(\theta)$ if and only if either $X_{i j}^{*}(\theta)>0$ or $X_{j i}^{*}(\theta)>0$, or equivalently, $(i, j) \in \mathcal{R}(\theta)$ if and only if $\left|U_{i}^{*}(\theta)-U_{j}^{*}(\theta)\right|>$ $\theta w_{i j}$. For $i \in V(\theta)$, let $\Delta_{i}^{(+)}(\theta)$ denote the number of edges $(k, i)$ with incoming flow (i.e., $\left.X_{k i}^{*}(\theta)>0\right)$; or formally, $\Delta_{i}^{(+)}(\theta):=\sum_{k} \mathbf{1}\left\{U_{k}^{*}(\theta)-U_{i}^{*}(\theta)>\theta w_{k i}\right\}$. Likewise, let $\Delta_{i}^{(-)}(\theta)$ denote the number of edges $(i, j)$ with outgoing flow (i.e., $X_{i j}^{*}(\theta)>0$ ); thus $\Delta_{i}^{(-)}(\theta):=\sum_{j} \mathbf{1}\left\{U_{i}^{*}(\theta)-U_{j}^{*}(\theta)>\right.$ $\left.\theta w_{i j}\right\}$. Define $\Delta_{i}(\theta):=\Delta_{i}^{(-)}(\theta)-\Delta_{i}^{(+)}(\theta)$, and $d_{i}(\theta)=$ $\sum_{j:\left|U_{i}-U_{j}\right|>\theta w_{i j}} a_{i j}=\sum_{j:(i, j) \in E(\theta)} a_{i j}$, where $a_{i j}:=w_{i j}^{-1}$ if $w_{i j}>0$, and $a_{i j}:=0$ if otherwise. Then from eq.(19), we see that the optimal $U_{i}^{*}(\theta)$ 's satisfy the following conditions:

$d_{i}(\theta) U_{i}^{*}(\theta)-\sum_{j:(i, j) \in E(\theta)} a_{i j} U_{j}^{*}(\theta)-\theta \Delta_{i}(\theta)= \begin{cases}1 & i=1 \\ 0 & 1<i<n,\end{cases}$

and $U_{n}^{*}(\theta)=0$.

We can rewrite eq.(26) more compactly in the matrix form:

$$
L(\theta) U^{*}(\theta)-\theta \Delta(\theta)=\mathrm{b} .
$$

Here $L(\theta):=\left[L_{i j}(\theta)\right]$ is the standard graph Laplacian matrix [8] of $\mathcal{R}(\theta)$ (with the adjacency matrix $A(\theta):=$ $\left.\left[a_{i j}\right], i, j \in V(\theta)\right)$, restricted to $V(\theta)-\{n\}$, namely, $L_{i i}(\theta):=$ $d_{i}(\theta)$, and $L_{i j}(\theta):=-a_{i j}, i, j \in V(\theta)-\{n\} . \Delta(\theta):=$ $\left[\Delta_{i}(\theta)\right]$, the vector consisting of $\Delta_{i}(\theta)$, and $\mathrm{b}=[1,0, \ldots, 0]^{T}$ is the vector corresponding to the right hand side of eq.(26). Since $\mathcal{R}(\theta)$ is connected, $L(\theta)$ is non-singular and thus

$$
U^{*}(\theta)=L^{-1}(\theta)(\theta \Delta(\theta)+\mathbf{b}) .
$$

Hence given $\theta$, we can explicitly solve for $U^{*}(\theta)$ using eq.(29). However, the definitions of both $L(\theta)$ and $\Delta(\theta)$ hinge on the routing graph $\mathcal{R}(\theta)=(V(\theta), E(\theta))$, which is itself defined assuming we know $X_{i j}^{*}(\theta)$ !

This circular dependency fortunately can be broken. From Theorem 1, we know that there exist only a finite sequence of routing graphs, $\mathcal{R}\left(\theta_{m}\right), 0 \leq m \leq M$, where $0=\theta_{0}<\theta_{1}<$ $\ldots<\theta_{M}$. In other words, for $\theta_{m} \leq \theta<\theta_{m+1}, 0 \leq m \leq M$ (and define $\left.\theta_{M+1}=\infty\right), \mathcal{R}(\theta)=\mathcal{R}\left(\theta_{m}\right)$. Hence if we know $\mathcal{R}\left(\theta_{m}\right)$, we can solve $U^{*}(\theta)$ for any $\theta_{m} \leq \theta<\theta_{m+1}$ and thus $X^{*}(\theta)$. This leads to the following recursive process for computing the routing continuum and $X^{*}(\theta)$ for all $\theta \geq 0$. 
Phase 1: from $\theta_{0}=0$ to $\theta_{1}$ :

When $\theta=0\left(=\theta_{0}\right), L(0)$ is simply the graph Laplacian of the original network $G$ (restricted to $V-\{n\}$ ). Then $U^{*}(0)=L^{-1}(0) \mathrm{b}$ is the optimal solution to the $L_{2}$-norm flow optimization, and $\mathcal{R}(0)$ is the "all-path" routing graph induced by the optimal $L_{2}$-norm flow $X^{*}(0)$.

Now consider any sufficient small $\theta>0$ (any $\theta<\theta_{1}$ would suffice) such that $\mathcal{R}(\theta)=\mathcal{R}(0)$ (thus $X_{i j}^{*}(\theta)>0$ for any $(i, j) \in \mathcal{R}(0)$. Hence $\Delta(\theta)=\Delta(0), L(\theta)=L(0)$, and $U^{*}(\theta)$ is given by

$$
U^{*}(\theta)=L^{-1}(0)(\theta \Delta(0)+\mathrm{b})=U^{*}(0)+\theta L^{-1}(0) \Delta(0) .
$$

From eq.(21) and eq.(30), if $U_{i}(\theta)-U_{j}(\theta)>\theta w_{i j}$,

$$
X_{i j}^{*}(\theta)=\frac{U_{i}^{*}(\theta)-U_{j}^{*}(\theta)}{w_{i j}}-\theta=X_{i j}^{*}(0)-\theta \alpha_{i j}(0),
$$

where $\alpha_{i j}(0)=1-\left(\beta_{i}(0)-\beta_{j}(0)\right) / w_{i j}$ is a constant, with $\beta_{i}(0)=\left[L^{-1}(0) \Delta(0)\right]_{i}$, if $i \in V-\{n\}$; and $\beta_{i}(0)=0$, if $i=n$. Eq.(30) shows that $U^{*}(\theta)$ is linear function of $\theta$, and for any edge $(i, j)$ where $X_{i j}^{*}(0)>0, X_{i j}^{*}(\theta)$ is also linear in $\theta$. Clearly, on edge $(i, j)$ with $\alpha_{i j}(0)>0$, the optimal flow $X_{i j}^{*}(\theta)$ decreases when $\theta$ increases; whereas on those with $\alpha_{i j}(0)<0$, the optimal flow $X_{i j}^{*}(\theta)$ increases $(\theta$ has no impact on those edges with $\left.\alpha_{i j}(0)=0\right)$. Hence we know precisely the (first) boundary condition, namely, the smallest positive $\theta$, when the first set of edges are to be truncated from $\mathcal{R}(0)$, namely, those where $X_{i j}^{*}(\theta)$ becomes 0 :

$$
\theta_{1}:=\min _{\langle i, j\rangle: \alpha_{i j}(0)>0}\left\{X_{i j}^{*}(0) / \alpha_{i j}(0)\right\} .
$$

Removing these edges yields the next routing graph $\mathcal{R}\left(\theta_{1}\right)$, for which $L\left(\theta_{1}\right)$ and $\Delta\left(\theta_{1}\right)$ can now be defined. Using eqs.(30) and (31), we can solve for the optimal solution, $U^{*}\left(\theta_{1}\right)$, and consequently, $X^{*}\left(\theta_{1}\right)$.

Phase 2: from $\theta_{k}$ to $\theta_{k+1}$ :

More generally, given $\mathcal{R}\left(\theta_{k}\right)$, and the corresponding optimal solutions, $U^{*}\left(\theta_{k}\right)$ and $X^{*}\left(\theta_{k}\right)$, we can solve for $U^{*}(\theta)$ and $X^{*}(\theta)$ for any $\theta_{k} \leq \theta<\theta_{k+1}$, using a similar argument. Again from eq.(29), with $L(\theta)=L\left(\theta_{k}\right)$ and $\Delta(\theta)=\Delta\left(\theta_{k}\right)$, we have

$$
\begin{aligned}
U^{*}(\theta) & =L^{-1}\left(\theta_{k}\right)\left(\theta \Delta\left(\theta_{k}\right)+\mathrm{b}\right) \\
& =U^{*}\left(\theta_{k}\right)+\left(\theta-\theta_{k}\right) L^{-1}\left(\theta_{k}\right) \Delta\left(\theta_{k}\right),
\end{aligned}
$$

and if $U_{i}(\theta)-U_{j}(\theta)>\theta w_{i j}$,

$$
X_{i j}^{*}(\theta)=\frac{U_{i}^{*}(\theta)-U_{j}^{*}(\theta)}{w_{i j}}-\theta=X_{i j}^{*}\left(\theta_{k}\right)-\theta \alpha_{i j}\left(\theta_{k}\right),
$$

where $\alpha_{i j}\left(\theta_{k}\right)=1-\left(\beta_{i}\left(\theta_{k}\right)-\beta_{j}\left(\theta_{k}\right)\right) / w_{i j}$ is a constant, with $\beta_{i}\left(\theta_{k}\right)=\left[L^{-1}\left(\theta_{k}\right) \Delta\left(\theta_{k}\right)\right]_{i}$, if $i \in V\left(\theta_{k}\right)-\{n\}$; and $\beta_{i}\left(\theta_{k}\right)=0$, if $i=n$. This gives us the next boundary, $\theta_{k+1}$, for the next set of links to be truncated (from $\mathcal{R}\left(\theta_{k}\right)$ ), where

$$
\theta_{k+1}=\min _{\langle i, j\rangle: \alpha_{i j}\left(\theta_{k}\right)>0}\left\{X_{i j}^{*}\left(\theta_{k}\right) / \alpha_{i j}\left(\theta_{k}\right)\right\}+\theta_{k} .
$$

Removing these edges from $\mathcal{R}\left(\theta_{k}\right)$ yields $\mathcal{R}\left(\theta_{k+1}\right)$, using which we can then solve for the optimal solutions, $U^{*}\left(\theta_{k+1}\right)$ and $X^{*}\left(\theta_{k+1}\right)$.

A pseudo-code algorithm for computing the boundary conditions $\theta_{m}$ 's, $0 \leq m \leq M$, is given in Algorithm 1, and for computing the optimal flow solution, $X^{*}(\theta)$, is given in Algorithm 2. Since each step of the recursive process involves solving a set of linear equations, the worse case complexity of which is $O\left(n^{3}\right)$, and $M$ is at most $|E|$ (the number of edges), the worst-case complexity of computing the entire routing continuum is $O\left(n^{3}|E|\right)$, or $O\left(n^{5}\right)$ in the worst case.

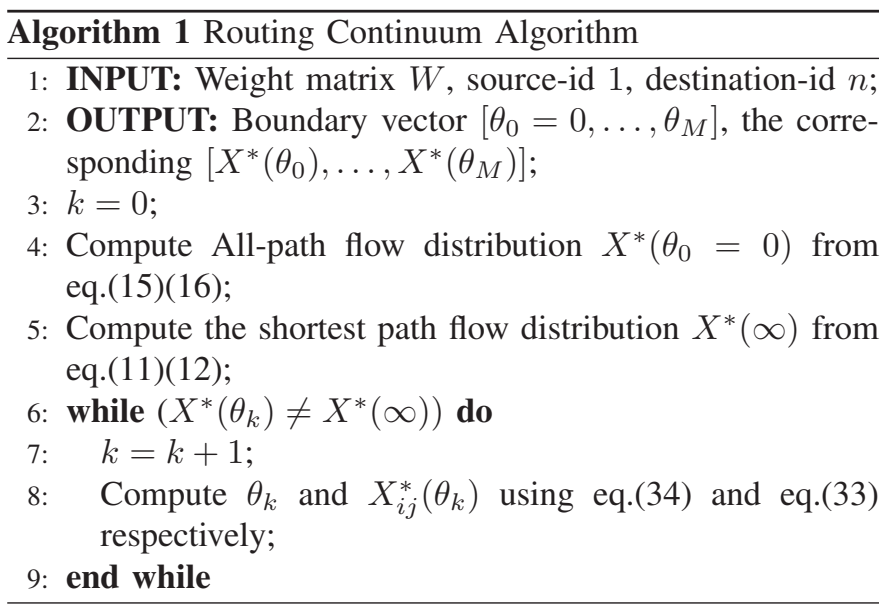

Algorithm 2 Solving the Optimal Flow Distribution $X^{*}(\theta)$, for a $\theta \geq 0$.

1: INPUT: $\left[\theta_{0}=0, \ldots, \theta_{M}\right],\left[X^{*}\left(\theta_{0}\right), \ldots, X^{*}\left(\theta_{M}\right)\right], \theta, W$, source-id 1 , destination-id $n$;

2: OUTPUT: Optimal flow distribution $X^{*}(\theta)$;

3: Search for the interval, such that $\theta \in\left[\theta_{k}, \theta_{k+1}\right)$;

4: Compute the $X^{*}(\theta)$ from eq.(33), for $\theta$.

\section{B. Numerical Analysis}

We now use two numerical examples to show how the routing continuum grows as the parameter $\theta$ changes. Fig. 1 shows an example topology, with three disjoint paths between the source 1 and the destination 5, and each link is with 1unit weight. As the parameter $\theta \geq 0$ increases, the longer paths $P 3=\{1 \rightarrow 3 \rightarrow 4 \rightarrow 5\}$ and $P 2=\{1 \rightarrow 2 \rightarrow 5\}$ are truncated gradually, and the shortest path $P 1=\{1 \rightarrow 5\}$ is obtained when $\theta$ increases to 1 . Fig. 2 shows the routing continuum, i.e. the optimal flow distributions at each $\theta$. We see that within the interval $\theta \in[0,0.4]$, the flows on the longer paths $P 2$ and $P 3$ get linearly redistributed to the shortest path $P 1$, and the longest path $P 3$ gets truncated when $\theta=0.4$. Then the flow of the second longest path $P 2$ keeps decreasing as $\theta$ increases, until the second boundary condition $\theta=1$ holds, where $P 2$ is truncated. During the routing evolution process, the network flows are always redistributed from longer paths to the shorter path, while increasing $\theta$. When 


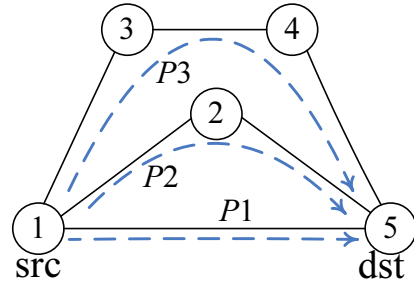

Fig. 1. Example 1 with uniform weight $w_{i j}=1$

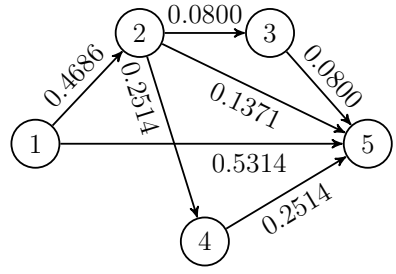

Fig. 5. Flow distribution with $\theta_{1}=0.0914$

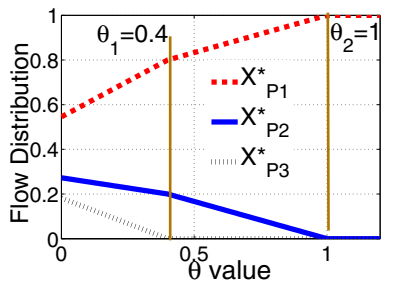

Fig. 2. Flow distribution evolution of graph Fig. 1

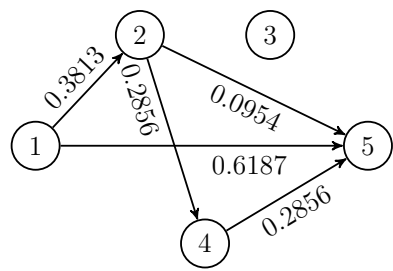

Fig. 6. Flow distribution with $\theta_{2}=0.2850$

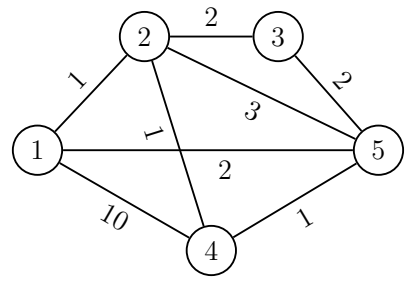

Fig. 3. Example 2: Weight Graph

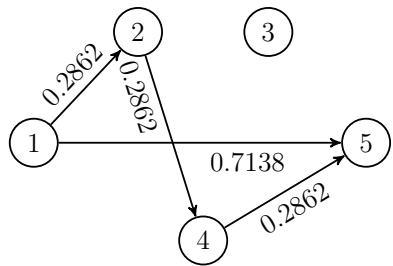

Fig. 7. Flow distribution with $\theta_{3}=0.5700$

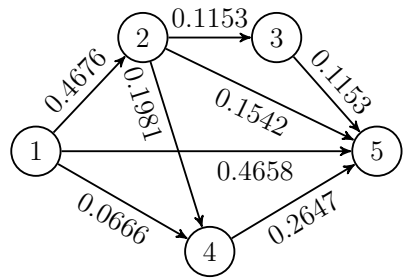

Fig. 4. Flow distribution, $\theta_{0}=0$

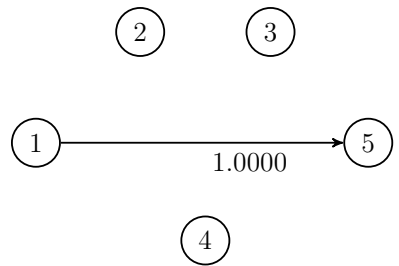

Fig. 8. Flow distribution with $\theta_{4}=2$ $\theta>1$, namely, the largest boundary condition, the routing solution is stabilized to the shortest path, i.e. $P 1$.

Fig. 3 shows another example with five connected nodes in the topology. Weights $w_{i j}$ 's are marked on the links. The flow initiates at source 1 and is removed from destination 5 . Fig. 4 Fig. 8 show the optimal flow distributions (marked on individual links) under five boundary conditions, $\left[\theta_{0}=0, \theta_{1}=\right.$ 0.0914, $\left.\theta_{2}=0.2850, \theta_{3}=0.5700, \theta_{4}=2\right]$, In Fig. $4\left(\theta_{0}=0\right)$, every link is active and follows the potential based "all-path" routing. Then, as $\theta$ increases to $\theta_{1}=0.0914$ (in Fig.5), link $(1,4)$ is truncated, and within the interval $\theta \in\left[0, \theta_{1}\right]$, only the flow on path $\{1 \rightarrow 4 \rightarrow 5\}$ decreases, and gets redistributed to other paths, because this path with total length 11 is the longest path in $\mathcal{P}(0)$, i.e. the "all-path" routing graph. Then, when $\theta$ increases to $\theta_{2}=0.2850$, the flows on links $(2,3)$ and $(3,5)$ are truncated, because these two links are on the second longest path $\{1 \rightarrow 2 \rightarrow 3 \rightarrow 5\}$, with path length 5. Similarly, when $\theta$ keeps increasing to $\theta_{3}$ and $\theta_{4}$, the rest two longer paths $\{1 \rightarrow 2 \rightarrow 5\}$ and $\{1 \rightarrow 2 \rightarrow 4 \rightarrow 5\}$ get removed, respectively, and only the shortest path $\{1 \rightarrow 5\}$ is left at last.

\section{Generalizations And Applications}

In this section, we present some extensions to the mixed $L_{1} / L_{2}$-norm network flow optimization, and briefly touch on their potential applications to traffic engineering and wireless sensor networks.

\section{A. Multiple Flows, Link/node Capacity Constraints and Traffic Engineering}

In the previous sections, for simplicity we have assumed a single flow of unit 1 from source node 1 to destination $n$. The formulation can be easily extended to accommodate multiple flows [21], [26], [30] between different source-destination pairs and with different units, as flows are additive on (links of) the network. Consider $K$ flows, where the $k$-th flow $X^{(k)}$ of $I^{(k)}$ units is routed from source node $s_{k}$ to destination node $t_{k}, 1 \leq k \leq K$. Thus each flow $X^{(k)}$ satisfies the following conservation constraints:

$$
\sum_{j:(i, j) \in E} X_{i j}^{(k)}-\sum_{l:(l, i) \in E} X_{l i}^{(k)}= \begin{cases}I^{(k)} & \text { if } i=s_{k}(\mathrm{Src}) \\ -I^{(k)} & \text { if } i=t_{k}(\mathrm{Dst}) \\ 0 & \text { if } i \neq s_{k}, t_{k}\end{cases}
$$

We use $\mathcal{F}_{k}$ to denote the collection of flows satisfying eq.(35). Then the mixed $L_{1} / L_{2}$-norm multi-flow optimization is given in eq.(36). It is not too hard to see that this problem can be decomposed into $K$ subproblems, each of which forms a single-flow mixed $L_{1}$ and $L_{2}$-norm optimization problem, and thus can be solved using the method presented before.

$$
\min _{\substack{X(k) \in \mathcal{F}_{k} \\ 1 \leq k \leq K}} \sum_{k=1}^{K} \sum_{i=1}^{n} \sum_{j=1}^{n}\left(w_{i j} X_{i j}^{(k)^{2}}+2 \theta w_{i j} X_{i j}^{(k)}\right)
$$

subject to $X_{i j}^{(k)} \geq 0,1 \leq i, j \leq n, 1 \leq k \leq K$.

In addition to having multiple flows (demands), many practical network flow problems, e.g., traffic engineering in a data network, also impose the link capacity constraints [11], [17]. Namely, given a network $G=(V, E)$, for each edge $(i, j) \in E$, let $C_{i j}\left(=C_{j i}\right)$ denote the link capacity. Then the total amount of flows on link $(i, j)$ cannot exceed $C_{i j}$. Given any set of $K$ flows, $X^{(k)} \in \mathcal{F}_{k}, 1 \leq k \leq K$, let $\alpha$ be a variable representing the maximum link utilization in the network, i.e., $\sum_{k} X_{i j}^{(k)} \leq \alpha C_{i j}$. Similar to [30], we consider the following maximum link utilization optimization and mixed $L_{1} / L_{2}$-norm flow optimization with link capacity constraints (where $\epsilon=\theta^{-1}$ ): 
Capacity Constrained Mixed Flow Optimization (Prime):

$$
\begin{aligned}
& \min _{\substack{X^{(k)} \in \mathcal{F}_{k} \\
1 \leq k \leq K}} \alpha+\sum_{k=1}^{K} \sum_{i=1}^{n} \sum_{j=1}^{n}\left(\frac{\epsilon}{2} w_{i j} X_{i j}^{(k)^{2}}+w_{i j} X_{i j}^{(k)}\right) \\
& \text { subject to } X_{i j}^{(k)} \geq 0,1 \leq k \leq K \text {; and } \\
& \sum_{k=1}^{K} X_{i j}^{(k)} \leq C_{i j} \alpha, 1 \leq i, j \leq n .
\end{aligned}
$$

Let $U_{i}^{(k)}$ be the Lagrange multipliers for the flow conservation constraints eq.(35), and $s_{i j}$ the Lagrange multipliers for the inequality constraints $\sum_{k=1}^{K} X_{i j}^{(k)}-C_{i j} \alpha \leq 0$. Then the dual problem is given by

Capacity Constrained Mixed Flow Optimization (Dual):

$$
\begin{aligned}
& \max _{U, \mathrm{~s}} \sum_{k=1}^{K} I^{(k)} U_{1}^{(k)} \\
& -\frac{1}{2} \sum_{k=1}^{K} \sum_{i=1}^{n} \sum_{j: U_{i}^{(k)}} \sum_{-U_{j}^{(k)}>w_{i j}+s_{i j}} \frac{\left(U_{i}^{(k)}-U_{j}^{(k)}-\left(w_{i j}+s_{i j}\right)\right)^{2}}{\epsilon w_{i j}}
\end{aligned}
$$

subject to $s_{i j} \geq 0, \sum_{i=1}^{n} \sum_{j=1}^{n} s_{i j} C_{i j}=1$, and $U_{n}^{(k)}=0$.

Let $\alpha^{*}$ and $X^{(k)^{*}}{ }_{i j}$ 's be the optimal solution to the primal problem and $U^{(k)^{*} \text { 's }}$ and $s_{i j}^{*}$ 's the optimal solution to the dual problem. Then by the complementary slackness, we have $X^{(k)^{*}}{ }_{i j}>0$ if and only if $U^{(k)^{*}}-U^{(k)^{*}} \geq w_{i j}+s_{i j}^{*}$; and furthermore, if $s_{i j}^{*}>0$, then $\sum_{k=1}^{K} X^{(k)^{*}}{ }_{i j}=C_{i j} \alpha^{*}$. The latter implies that any link $(i, j) \in E$ with $s_{i j}^{*}>0$ is a "bottleneck" link where the (optimal) maximum link utilization is attained. We see that on a bottleneck link $(i, j)$, if $X^{(k)}{ }_{i j}^{*}>0$, then $X^{(k)_{i j}^{*}}=\left(U^{(k)_{i}^{*}}-U^{(k)_{j}^{*}}-\left(w_{i j}+s_{i j}^{*}\right)\right) /\left(\epsilon w_{i j}\right)$; whereas on a non-bottleneck link (i.e., $s_{i j}^{*}=0$ ), if $X^{(k)^{*}}{ }_{i j}>0$, then $X^{(k)_{i j}^{*}}=\left(U^{(k)_{i}^{*}}-U^{(k)_{j}^{*}}-w_{i j}\right) /\left(\epsilon w_{i j}\right)$.

Comparing this with the optimal flow solutions to the mixed $L_{1} / L_{2}$-norm without the capacity constraints, an additional $s_{i j}^{*} /\left(\epsilon w_{i j}\right)$ amount is reduced from each flow $X^{(k)}{ }_{i j}^{*}$ on the bottleneck links $(i, j)$. Intuitively, it is as if the weights on the bottleneck links were replaced with $w_{i j}^{\prime}=w_{i j}+s_{i j}^{*}$ to discourage and shift away flows on the bottleneck links. In fact, suppose $s_{i j}^{*}$ 's are known a priori. We can convert the network flow optimization eq.(37) with link capacity constraints to one (without link capacity constraints) as eq.(36), where $w_{i j}$ 's in the $L_{1}$-norm term are replaced by $w_{i j}^{\prime}:=w_{i j}+s_{i j}^{*}$ 's, but not those in the $L_{2}$-norm term. This yields an example of network flow optimization with heterogenous $L_{1} / L_{2}$ costs to be discussed in the next subsection. Intuitively, this implies that the optimal flow with link capacity constraints that minimizes overall maximum link utilization is the one that discourages the usage of bottleneck links by increasing the $\left(L_{1}\right)$ link costs on these links and thus shifting flows away from them.

Finally, for each $\epsilon\left(=\theta^{-1}\right)$, we can use the optimal solution $\alpha^{*}(\theta)$ to eq.(37) to determine the best trade-offs between using shorter paths and longer paths, namely, the best routing graph
$\mathcal{R}(\theta)$ which minimizes the overall network link utilization $\alpha(\theta)$ among any choice of $\theta \geq 0$ :

$$
\theta^{*}:=\operatorname{argmin}_{\theta \geq 0} \alpha^{*}(\theta) .
$$

In general, with the link capacity constraints, finding the optimal $\theta^{*}$ requires search in the solution space, $\left\{\theta: \alpha^{*}(\theta)\right\}$. On the other hand, assuming that $w_{i j}$ 's are fixed, we can find the optimal $\theta^{*}$ in polynomial time by first computing the entire routing continuum using Algorithms 1 and 2, and then calculating the corresponding maximum link utilization $\alpha^{*}(\theta):=\max _{(i, j)}\left\{\sum_{k} X^{(k)_{i j}^{*}} / C_{i j}\right\}$ for each $\theta>0$. Thus with respect to a fixed set of link weights $w_{i j}$ 's, the routing graph $\mathcal{R}\left(\theta^{*}\right)$ yields the best trade-offs in usage of shorter and long paths: it minimizes the overall network utilization among all routing graphs.

Moreover, practical network flow problems, e.g., routing in bandwidth constrained wireless networks, may involve node capacity constraints [5], [10], [32], where for node $i \in V$, with node capacity $C_{i}$, the total amount of flows going through node $i$ cannot exceed $C_{i}$. For a set of $K$ flows, $X^{(k)} \in \mathcal{F}_{k}$, $1 \leq k \leq K, \sum_{k} \sum_{j} X_{i j}^{(k)} \leq \xi C_{i}$ holds true, where $\xi$ is the maximum node capacity utilization in the network. While considering node capacity constrained mixed $L_{1} / L_{2}$-norm flow optimization problem, similar results can be obtained as the link capacity constrained $L_{1} / L_{2}$-norm optimization problem. We omit the details here for brevity.

\section{B. Flow Optimization with Heterogeneous $L_{1} / L_{2}$ Link Weights}

We consider the following generalization where $L_{1}$-norm and $L_{2}$-norm have different sets of link weights, $w_{i j}$ 's and $r_{i j}$ 's:

Flow Optimization with Heterogeneous $L_{1} / L_{2}$ Weights (Prime):

$$
\begin{aligned}
& \min _{\substack{X^{(k)} \in \mathcal{F}_{k} \\
1 \leq k \leq K}} \sum_{k=1}^{K} \sum_{i=1}^{n} \sum_{j=1}^{n}\left(r_{i j} X_{i j}^{(k)^{2}}+2 \theta w_{i j} X_{i j}^{(k)}\right), \\
& \text { subject to } X_{i j}^{(k)} \geq 0,1 \leq k \leq K .
\end{aligned}
$$

We have already seen one instance of such generalization in the application of traffic engineering with link/node capacity constraints. Another application arises more naturally in wireless sensor networks, where deciding on the best strategies hinge on trading off different cost considerations [18], e.g., transmission latency as well as energy consumption - the latter is important, for example, to maximize the sensor network life time, where it is shown in [19] that potential-based routing using $L_{2}$-norm maximizes the network life time. Let $w_{i j}$ 's denote the per-hop transmission latency, and $r_{i j}$ 's be the transmission energy costs. Then, eq.(41) represents the mixed $L_{1} / L_{2}$-norm network flow optimization problem with heterogeneous $L_{1} / L_{2}$ link weights. The dual problem can be formulated as follows: 
Flow Optimization with Heterogeneous $L_{1} / L_{2}$ Weights (Dual):

$$
\begin{aligned}
& \max _{U, \mathrm{~s}} \sum_{k=1}^{K} I^{(k)} U_{1}^{(k)}-\frac{1}{2} \sum_{k=1}^{K} \sum_{i=1}^{n} \sum_{j:(i, j) \in E^{(k)}(\theta)} \frac{\left(U_{i}^{(k)}-U_{j}^{(k)}-\theta w_{i j}\right)^{2}}{r_{i j}} \\
& \text { subject to } U_{n}^{(k)}=0,1 \leq k \leq K,
\end{aligned}
$$

where $E^{(k)}(\theta)$ is the edge set, link $(i, j) \in E^{(k)}(\theta)$ if and only if $U_{i}^{(k)}-U_{j}^{(k)}>\theta w_{i j}$. Let $X_{i j}^{(k)^{*}}$ 's and $U^{(k)^{*}}{ }_{i}$ 's be the optimal solution to the primal and dual problems, respectively. By complementary slackness, we have $X^{(k)_{i j}^{*}}=$ $\left(U^{(k)_{i}^{*}}-U^{(k)^{*}}-\theta w_{i j}\right) / r_{i j}>0$ if and only if $U^{(k)_{i}^{*}}-$ $U^{(k)_{j}^{*}}>\theta w_{i j}$. Using this relation, we can generalize Lemma 2 as below:

$$
\theta \sum_{\langle i, j\rangle \in P^{(k)}} w_{i j}<U_{1}^{(k)^{*}}(\theta) \leq \theta \sum_{\langle i, j\rangle \in Q^{(k)}} w_{i j}+\sum_{\langle i, j\rangle \in Q^{(k)}} r_{i j},
$$

where $P^{(k)}$ is a routing path with nonzero flow $X^{(k)^{*}}$ from source $s_{k}$ to destination $t_{k}$ (i.e., $P^{(k)} \in G_{X^{(k)}}$ ), whereas $Q^{(k)}$ is an arbitrary (simple) path in the network $G$ from source $s_{k}$ to destination $t_{k}$. For any given $\theta \geq 0$ and $1 \leq k \leq K$, using eq.(42) we can again characterize all paths in the routing graph $\mathcal{R}^{(k)}(\theta)$ - the routing graph induced by $X^{(k)^{*}}(\theta)$ : for any $P \in \mathcal{R}^{(k)}(\theta)$, its path length, $|P|<L_{\min , 1}^{(k)}+\theta^{-1} L_{\max , 2}^{(k)}$, where $L_{\min , 1}^{(k)}:=\min _{Q^{(k)}} \sum_{\langle i, j\rangle \in Q^{(k)}} w_{i j}$ is the path length of the shortest (in terms of $L_{1}$ link weights ) paths from $s_{k}$ to $t_{k}$, and $L_{\max , 2}^{(k)}:=\max _{Q^{(k)} \in \mathcal{R}^{(k)}(0)} \sum_{\langle i, j\rangle \in Q^{(k)}} r_{i j}$ is the the path length of the longest (in terms of $L_{2}$ link weights) paths in the routing graph $\mathcal{R}^{(k)}(0)$, the routing graph induced by the optimal $L_{2}$-norm flow $X^{(k)}(0)$. Therefore, we can establish a generalized routing continuиm theorem analogous to Theorem 1, yielding a finite sequence of routing graphs, $\mathcal{R}^{(k)}\left(\theta_{m}^{(k)}\right)$ 's. Furthermore, the boundary conditions for $\theta_{m}^{(k)}$, s can be precisely characterized using a similar iterative process as presented in Section IV-A, and Algorithms 1 and 2 can be analogously generalized to compute the entire routing continuum and $\left\{X^{(k)^{*}}(\theta), 1 \leq k \leq K\right\}$ for all $\theta>0$. We omit the details here for brevity.

\section{RELATED WORK AND DISCUSSION}

Routing in networks has been extensively studied under practical settings, with a literature too vast to cite completely. Here we will mention a few that are most relevant. For example, the authors in [20] propose an optimization model for QoS routing protocol design with multiple $L_{1}$ norm performance objectives, where the objectives are linearly combined with tunable parameter. In the context of traffic engineering in IP data networks, the authors in [12] show that given a set of traffic demands, optimizing the link weights in a network (assuming shortest-path routing) is NP-hard, and develop heuristics. The authors in [31] propose a new linkstate routing protocol PEFT that goes beyond shortest paths by allowing longer paths and splitting traffic over multiple paths with an exponential penalty on longer paths. Via convex optimization, the authors show PEFT achieves optimal traffic engineering. The studies in [2], [3], [9], [24] analyze the tradeoffs between shortest path routing and multi-path routing in both wired and wireless network settings.

Different from earlier works, which focus on routing protocol designs for specific (wired/wireless) network scenarios, our work studies routing from a more general and theoretical perspective. It is partly inspired by the finding in [30], where motivated by traffic engineering in IP networks, the authors show that shortest path routing results from the optimal flow minimizing the $L_{1}$-norm in a network. In contrast, the optimal flow minimizing the $L_{2}$-norm in a network and its connection to currents in resistive electrical networks (and random walks on a graph) are well-known (see [16] and references thereof); it leads to the potential-based, "all-path" (or stochastic) routing that has been applied in wireless sensor networks, e.g., to maximize network life time [19], or to minimize state maintenance [7]. Our work generalizes these earlier results to show that using the mixed $L_{1} / L_{2}$-norm flow optimization, we can construct the entire routing continuum from the shortestpath to all-path, with routing graphs consisting of paths of increasing path lengths.

In a broader context, the mixed $L_{1} / L_{2}$ optimization formulation has been widely used, e.g., in the classical LASSO problems [28], namely, the least square optimization problems with a $L_{1}$-norm penalty term, and more recently, in compressive sensing [6], [29]. It is therefore well-known that the $L_{1}$ norm penalty forces the least-square solution, $X^{*}$, to meet certain sparsity constraints, i.e., $\left\|X^{*}\right\|_{1} \leq \epsilon$. Compared with LASSO and compressive sensing settings, our setting has a set of additional flow conservation constraints - these are what makes the problem unique and leads to solutions that have interesting interpretations and consequences, where the solutions to the more general LASSO and compressive sensing settings may not have, apart from the sparsity of the solutions.

Indeed, Lemma 2 in our paper shows that our results can be interpreted in terms of the "sparsity" of the solutions also: the optimal flow solution $X^{*}(\theta)$ to the mixed $L_{1} / L_{2}$-norm flow optimization leads to a sparser routing graph, where the path length of routes used for routing the optimal flow from a source to a destination can not be $\left(1+\theta^{-1}\right)$ longer than the shortest paths. More surprising and interesting is that we can generate the entire routing continuum from the mixed $L_{1} / L_{2}-$ norm flow optimization. The flow conservation constraints in fact play a key role here: it leads to the duality of the optimal flows, $X^{*}(\theta)$, a function defined on the edges of a network, and the optimal (generalized) potential functions, $U^{*}(\theta)$, a function defined on the nodes of a network. This allows us to solve $U^{*}(\theta)$ through a set of linear equations, and yields an efficient process to compute the entire routing continuum and the optimal flow $X^{*}(\theta)$ for any $\theta \geq 0$. Last but not the least, we remark that although we only focus on network routing in this paper, we believe that our results can be applied to many other applications where the problems can be cast in terms of flows in a network. 


\section{CONCLUSION}

In this paper, we have formulated the network routing problem as flow optimization problem in a network with mixed $L_{1} / L_{2}$-norms. Using this formulation, we established a surprising result: the routing graphs induced by the optimal flow solutions span the entire routing continuum from the shortest-path to all-path routing. Using the duality theory, we also developed an efficient iterative process for computing the entire routing continuum and optimal flow solutions $X^{*}(\theta)$ for any $\theta \geq 0$. The basic theory is further extended to account for multiple flows (traffic demands), link capacity constraints and heterogeneous $L_{1} / L_{2}$ link weights, with applications to traffic engineering and wireless sensor networks.

As part of future work, we will apply the routing continuum theory to the study of network robustness, both in terms of understanding the inherent robustness of various network topologies and as well as in the design of more resilient routing strategies. Going beyond the telecommunication and computer networks, we are also interested in applying and generalizing the theory to complex network analysis in other settings, e.g., social networks. For instance, given the optimal flow distribution $X^{*}(\theta)$ for a particular $\theta$, the total flow amount through a node $i$, averaged over all source destination pairs, naturally leads to a generalized centrality measure of node in a graph, which subsumes the shortest path betweenness [13], [14], [23] and random walk betweenness [22] as two special cases. Moreover, the optimal "voltage" $U_{1}^{*}(\theta)$ at the source node 1 yields a generalized distance measure for node pair $(1, n)$, that takes the effective resistance distance [27] as a special case, i.e., $U_{1}^{*}(0)$.

\section{REFERENCES}

[1] G. R. Ash. Dynamic Routing in Telecommunications Networks. McGraw-Hill Professional, 1st edition, 1997.

[2] C. L. Barrett, S. J. Eidenbenz, L. Kroc, M. V. Marathe, and J. P. Smith. Probabilistic multi-path vs. deterministic single-path protocols for dynamic ad-hoc network scenarios. In Proceedings of the 2005 ACM symposium on Applied computing, SAC '05, pages 1166-1173, New York, NY, USA, 2005. ACM.

[3] A. Basu, A. Lin, and S. Ramanathan. Routing using potentials: a dynamic traffic-aware routing algorithm. In SIGCOMM '03: Proceedings of the 2003 conference on Applications, technologies, architectures, and protocols for computer communications, pages 37-48, New York, NY, USA, 2003. ACM Press.

[4] S. Biswas and R. Morris. Exor: opportunistic multi-hop routing for wireless networks. In SIGCOMM '05: Proceedings of the 2005 conference on Applications, technologies, architectures, and protocols for computer communications, pages 133-144, New York, NY, USA, 2005. ACM.

[5] T. Bonald, M. Jonckheere, and A. Proutiére. Insensitive load balancing. In Proceedings of the joint international conference on Measurement and modeling of computer systems, SIGMETRICS '04/Performance '04, pages 367-377, New York, NY, USA, 2004. ACM.

[6] E. J. Candes and M. B. Wakin. An Introduction To Compressive Sampling. IEEE Signal Processing Magazine, 25(2):21-30, March 2008.

[7] C.-K. Chau and P. Basu. Exact analysis of latency of stateless opportunistic forwarding. In INFOCOM '09: Proceedings of the 28th IEEE Conference on Computer Communications, Rio de Janeiro, Brazil, 2009. IEEE.

[8] F. R. K. Chung and S. T. Yau. Discrete green's functions. Journal of Combinatorial Theory, Series A, pages 191-214, 2000.

[9] I. Cidon, R. Rom, and Y. Shavitt. Analysis of multi-path routing. IEEE/ACM Transactions on Networking., 7:885-896, December 1999.
[10] A. V. Donati, R. Montemanni, L. M. Gambardella, and A. E. Rizzoli. Integration of a robust shortest path algorithm with a time dependent vehicle routing model and applications. In Computational Intelligence for Measurement Systems and Applications, 2003. CIMSA '03. 2003 IEEE International Symposium on, 2003.

[11] W. Fisher, M. Suchara, and J. Rexford. Greening backbone networks: reducing energy consumption by shutting off cables in bundled links. In Proceedings of the first ACM SIGCOMM workshop on Green networking, Green Networking '10, pages 29-34, New Delhi, India, 2010. ACM.

[12] B. Fortz and M. Thorup. Increasing internet capacity using local search. Computational Optimization and Applications, 29(1):13-48, 2004.

[13] L. C. Freeman. A set of measures of centrality based on betweenness. Sociometry, 40(1):35-41, March 1977.

[14] L. C. Freeman. Centrality in social networks conceptual clarification. Social Networks, 1(3):215-239, 1979.

[15] D. Ganesan, R. Govindan, S. Shenker, and D. Estrin. Highly-resilient, energy-efficient multipath routing in wireless sensor networks. $S I G$ MOBILE Mobile Computing and Communications Review, 5(4):11-25, October 2001.

[16] F. P. Kelly. Network routing. Philosophical Transactions of the Royal Society, A(337):343-367, 1991.

[17] L. Li, M. Thottan, B. Yao, and S. Paul. Distributed network monitoring with bounded link utilization in ip networks. In INFOCOM '03: Proceedings of the 22th IEEE Conference on Computer Communications, pages 1189-1198, Hong Kong, China, 2003. IEEE.

[18] Y. Li and Z.-L. Zhang. Random walks on digraphs: A theoretical framework for estimating transmission costs in wireless routing. In INFOCOM '10: Proceedings of the 29th IEEE Conference on Computer Communications, San Diego, USA, 2010. IEEE.

[19] H. Liu, Z.-L. Zhang, J. Srivastava, and V. Firoiu. Pwave: A multi-source multi-sink anycast routing framework for wireless sensor networks. In NETWORKING '07: Proceedings of the 6th International IFIP-TC6 Networking, Atlanta, GA, USA, 2007. IFIP.

[20] P. V. Mieghem and L. Vandenberghe. Trade-off curves for qos routing. In Infocom '06: Proceedings of the 25th Annual Joint Conference of the IEEE Computer and Communications Societies, Barcelona, Catalunya, Spain, 2006. IEEE.

[21] M. Neely, E. Modiano, and C. Rohrs. Dynamic power allocation and routing for time varying wireless networks. In INFOCOM '03: Proceedings of the 22th IEEE Conference on Computer Communications, Hong Kong, China, 2003.

[22] M. E. J. Newman. A measure of betweenness centrality based on random walks. Social Networks, 27(1):39-54, January 2005.

[23] M. E. J. Newman. Networks: An Introduction. Oxford University Press, 1st edition, 2010.

[24] P. Pham. Performance analysis of reactive shortest single-path and multipath routing mechanism with load balance. In INFOCOM 2003. 22nd IEEE International Conference on Computer Communications, 2003.

[25] L. Popa, C. Raiciu, I. Stoica, and D. Rosenblum. Reducing congestion effects in wireless networks by multipath routing. In ICNP'06: IEEE International Conference on Network Protocols, pages 96-105, Los Alamitos, CA, USA, 2006. IEEE.

[26] P. Purkayastha and J. S. Baras. An optimal distributed routing algorithm using dual decomposition techniques. Communications in Information and Systems, 8(3):277-302, 2008.

[27] G. Ranjan and Z.-L. Zhang. Geometry of complex networks and structural centrality. Technical report, Techincal Report, CS, UMN, http://www.cs.umn.edu/ granjan, 2010.

[28] R. Tibshirani. Regression shrinkage and selection via the lasso. Journal of the Royal Statistical Society (Series B), 58:267-288, 1996.

[29] Y. Tsaig and D. L. Donoho. Compressed sensing. IEEE Transactions on Information Theory, 52:1289-1306, 2006.

[30] Y. Wang, Z. Wang, and L. Zhang. Internet traffic engineering without full mesh overlaying. In Infocom '01: Proceedings of the 20th Annual Joint Conference of the IEEE Computer and Communications Societies, Anchorage, Alaska, USA, 2001. IEEE.

[31] D. Xu, M. Chiang, and J. Rexford. Link-state routing with hop-by-hop forwarding can achieve optimal traffic engineering. In INFOCOM 2008. 27th IEEE International Conference on Computer Communications, pages 466-474, Phoenix, AZ, USA, 2008. IEEE.

[32] G. Zussman and A. Segall. Energy efficient routing in ad hoc disaster recovery networks. In INFOCOM '03: Proceedings of the 22th IEEE Conference on Computer Communications, Hong Kong, China, 2003. 